\title{
An Equivalent Model of Corrugated Structures
}

\section{Yu-Qian LI ${ }^{2}$, Jia-Yu WU ${ }^{1, a,}{ }^{*}$, Hao-Wei GU ${ }^{1}$, Zong-Yong $\mathrm{CHEN}^{2}$, Xiao-Bing SHI ${ }^{2}$, Hong YUAN ${ }^{1, b}$,}

${ }^{1}$ MOE Key Laboratory of Disaster Forecast and Control in Engineering, Institute of Applied Mechanics, Jinan University, Guangzhou 510632, China

${ }^{2}$ GuizhouNATURALL Technology Co., Ltd

a1191625174@qq.com, btyuanhong.jnu.edu.cn

${ }^{*}$ Corresponding author

Keywords: Corrugated Structures, Orthotropic Plate, Finite Element Method, Modal Analysis.

\begin{abstract}
Corrugated structures have wide application in engineering. The corrugated structure is replaced by an orthotropic plate with equivalent stiffness properties in this paper. After equivalent stiffnesses are obtained, deflections and modals of simply supported orthotropic plates can be analyzed theoretically, which are compared with finite element solutions modeled with corrugated structures. Finally, it is clearly that corrugated structures can be replaced by an orthotropic plate whit equivalent stiffness in deflection and modal analysis.
\end{abstract}

\section{Introduction}

Over last decades, corrugated structures have wide application in civil, aerospace, naval and automotive engineering, which is used as sensing elements, fiberboards, folded roofs, container walls, sandwich plate cores, bridge decks, ship panels, etc. The main mechanical property of corrugated structures is high specific stiffness, especially under bending.

When the dimensions of corrugated structure are much larger than the period of the corrugations, a homogenisation-based analytical model could be used for any corrugation shape, in which the corrugated structure is replaced by an orthotropic plate with equivalent stiffness properties. Briassoulis $^{[1]}$ and McFarland ${ }^{[2]}$ investigated the equivalent bending stiffness with sinusoidal and rectangular corrugations, respectively. Samanta and Mukhopadhyay performed the static and dynamic analyses of trapezoidal corrugated structures by considering both extensional and bending stiffness $^{[3]}$. Yokozekiet al. analyzed the mechanical properties of corrugated laminates made from carbon epoxy composites theoretically and experimentally ${ }^{[4]}$. Peng et al. investigated the equivalent elastic properties of sinusoidal and trapezoidal corrugated plates by means of a mesh-free Galerkin method $^{[5]}$. Liew et al. used this method for the geometrically nonlinear analysis of corrugated plates. Both the equivalent extensional and bending properties were employed in the analyses ${ }^{[6,7]}$. Winkler and Kress derived accurate analytical expressions of equivalent orthotropic plate for circular corrugations ${ }^{[8-10]}$. Xia et al. replaced the corrugated structure by an orthotropic plate, based on the equivalent energy and force properties ${ }^{[11]}$. Giorgio Bartolozzi et al. investigated corrugated structures with any shapes by curved beam theories, and obtained equivalent Elasticity modulus and Poisson's ratio $^{[12]}$. Ye et al. summarized the equivalent model of corrugated structures in the past fifty years, and present their own equivalent models with poor theoretical derivation ${ }^{[13]}$.

This paper replaces the corrugated structure by an orthotropic plate with equivalent stiffness properties. Deflections and modals of simply supported orthotropic plates can be analyzed orthotropic plates theoretically, which are compared with finite element solutions modeled with corrugated structures. 


\section{Equivalent Stiffness}

The corrugated structures are generated from a periodic shape in the $\mathrm{XZ}$ plane that is extruded in the Y direction to produce a structure, as seen in Fig.1.

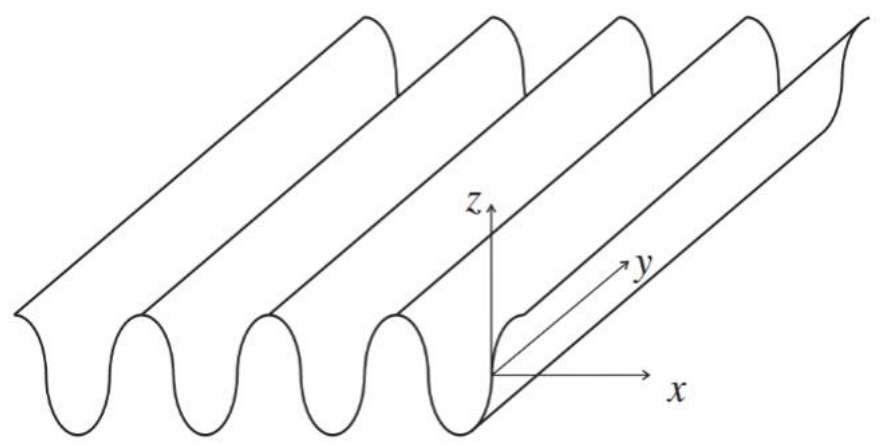

(a)

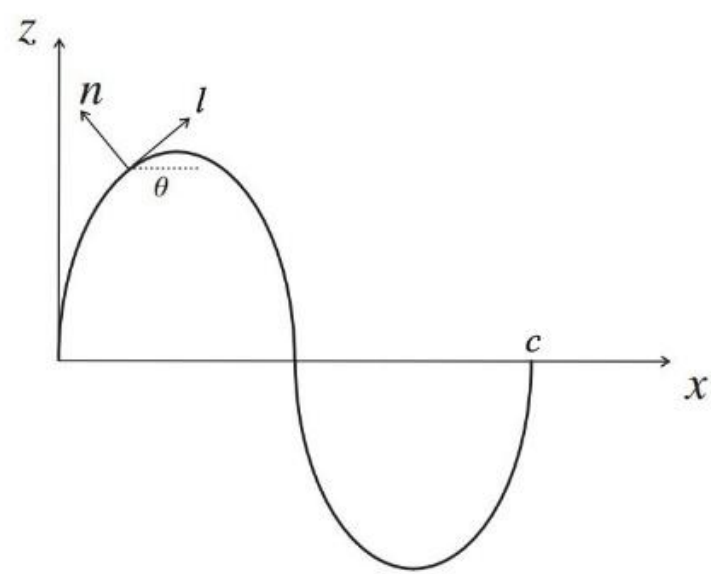

(b)

Fig.1 Definition of the Coordinate Systems

The five most common corrugated structures were discussed included trapezoidal, rectangular, toothed, sinusoidal and circular corrugations, as shown in Fig.2.

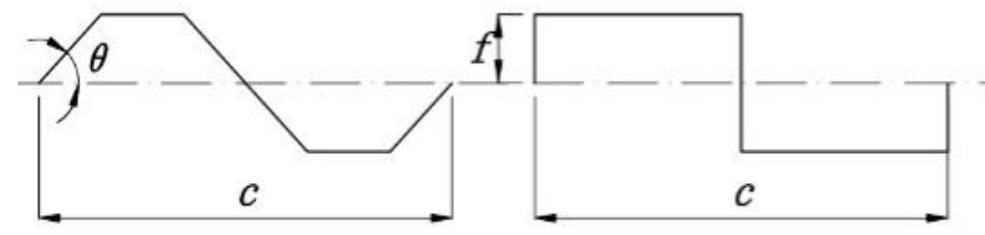

(a)

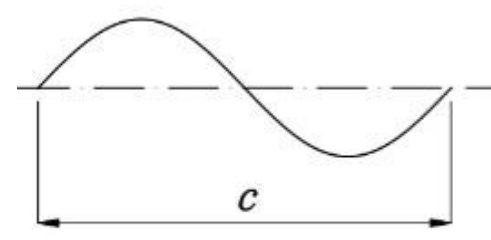

(d) (b)

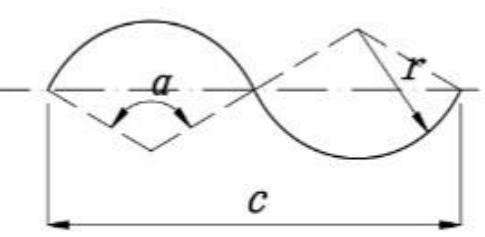

(e)

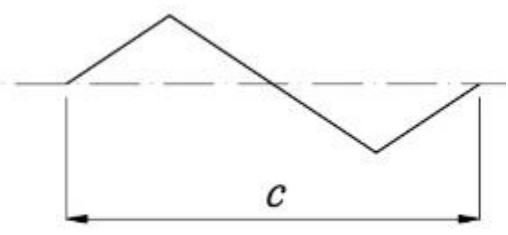

(c)

(a) Trapezoidal; (b) Rectangular; (c) Toothed; (d) Sinusoidal; (e) Circular

Fig. 2 The Most Common Corrugated Structures 
In order to have a better understanding of extension stiffness and bending stiffness of corrugated structures, which replaced by orthotropic plates, ANSYS FEA simulations are implemented to simulate the mechanical behaviors of the five most corrugated structures, which modeled with elastic shell element SHELL63.

Orthotropic structures are replaced by orthotropic plates. If coupling stiffnesses $\boldsymbol{B}$ is ignored, constitutive equations of equivalent orthotropic plates can be written as

$$
\left\{\begin{array}{l}
\overline{N_{x}} \\
\overline{N_{y}} \\
\overline{N_{x y}} \\
\overline{M_{x}} \\
\overline{M_{y}} \\
\overline{M_{x y}}
\end{array}\right\}=\left[\begin{array}{cccccc}
\overline{A_{11}} & \overline{A_{12}} & 0 & 0 & 0 & 0 \\
\overline{A_{12}} & \overline{A_{22}} & 0 & 0 & 0 & 0 \\
0 & 0 & \overline{A_{66}} & 0 & 0 & 0 \\
0 & 0 & 0 & \overline{D_{11}} & \overline{D_{12}} & 0 \\
0 & 0 & 0 & \overline{D_{12}} & \overline{D_{22}} & 0 \\
0 & 0 & 0 & 0 & 0 & \overline{D_{66}}
\end{array}\right]\left\{\begin{array}{l}
\overline{\varepsilon_{x}} \\
\overline{\varepsilon_{y}} \\
\frac{\gamma_{x y}}{\overline{\kappa_{x}}} \\
\overline{\kappa_{y}} \\
\overline{\kappa_{x y}}
\end{array}\right\}
$$

From Eq. (1), if one of strains is maintained 1, and the rest are 0 , the value of the force equals the value of responding stiffness. The equivalent stiffnesses are shown in Table 1 , where $I_{s y}$ is inertia moment relative to the neutral axis (X axis), and $s$ and $t$ are the corrugation length and the thickness of corrugated structure, respectively.

Tab.1 Equivalent Extension Stiffness and Bending Stiffness of Corrugated Structures

\begin{tabular}{|c|c|c|c|}
\hline & Extension stiffness & & Bending stiffness \\
\hline$\overline{A_{11}}$ & $\frac{E c t^{3}}{48 \int_{0}^{\frac{c}{4}} z^{2}(x) \sqrt{1+z^{\prime}(x)^{2}} d x}$ & $\overline{D_{11}}$ & $\frac{c}{s} \frac{E t^{3}}{12}$ \\
\hline$\overline{A_{12}}$ & $v \overline{A_{11}}$ & $\overline{D_{12}}$ & $v \overline{D_{11}}$ \\
\hline$\overline{A_{22}}$ & $\frac{E t}{c}$ & $\overline{D_{22}}$ & $\frac{E I_{s y}}{c}$ \\
\hline$\overline{A_{66}}$ & $\frac{c}{s} \frac{E t}{2(1+v)}$ & $\overline{D_{66}}$ & $\frac{s}{c} \frac{E t^{3}}{24(1+v)}$ \\
\hline
\end{tabular}

In the numerical examples, the material properties and the geometry parameters are $E=21 \mathrm{GPa}$, $v=0.3, b=c=0.1 \mathrm{~m}, f=0.015 \mathrm{~m}, t=0.004 \mathrm{~m}$. Trapezoidal corrugated structure with $\theta=45^{\circ}, s=0.12485 \mathrm{~m}$; Rectangular corrugated structure is a particular case of trapezoidal corrugated structures when $\theta=90^{\circ}$, with $s=0.16 \mathrm{~m}$; Toothed corrugated structure is also a particular case of trapezoidal corrugated structures when bottom side, with $\theta=30.964^{\circ}, s=0.11662 \mathrm{~m}$; Sinusoidal corrugated structure with the curve $z(x)=0.015 \sin (20 \pi x) \mathrm{m}, s=0.11945 \mathrm{~m}$; Circular corrugated structure with $r=0.028333 \mathrm{~m}$, $\alpha=123.855^{\circ}, s=0.1225 \mathrm{~m}$. Theoretical value and FEA value equivalent stiffnesses of the five most common corrugated structures are listed in Table 2.

Based on Table 2, bending stiffness along the corrugations direction $\overline{D_{11}}$ is much smaller than that vertical the corrugations direction $\overline{D_{22}}$. Additionally, both theoretical results and FEA results $\overline{A_{22}}$, $\overline{A_{66}}, \overline{D_{22}}$ and $\overline{D_{66}}$ are fairly close; the error $\overline{A_{11}}, \overline{A_{12}}, \overline{D_{11}}$ and $\overline{D_{12}}$ is about $7 \%-9 \%$. 
Tab.2 Equivalent Stiffness of the Five Most Common Corrugated Structures

\begin{tabular}{|c|c|c|c|c|c|c|}
\hline & & Trapezoidal & Rectangular & Toothed & Sinusoidal & Circular \\
\hline \multirow{3}{*}{$\begin{array}{c}\overline{A_{11}} \\
(\mathrm{MN} / \mathrm{m})\end{array}$} & Theoretical & 0.729 & 0.415 & 1.28 & 0.904 & 0.7844 \\
\hline & FEM & 0.784 & 0.446 & 1.37 & 0.971 & 0.8438 \\
\hline & Error $(\%)$ & 7.02 & 6.95 & 6.46 & 6.89 & 7.04 \\
\hline \multirow{3}{*}{$\begin{array}{c}\overline{A_{12}} \\
(\mathrm{MN} / \mathrm{m})\end{array}$} & Theoretical & 0.219 & 0.125 & 0.38 & 0.2712 & 0.2353 \\
\hline & FEM & 0.235 & 0.134 & 0.41 & 0.2915 & 0.2534 \\
\hline & Error $(\%)$ & 5.96 & 6.72 & 6.76 & 6.96 & 7.14 \\
\hline \multirow{3}{*}{$\begin{array}{c}\overline{A_{22}} \\
(\mathrm{MN} / \mathrm{m})\end{array}$} & Theoretical & 104.88 & 134.4 & 98 & 100.338 & 102.9 \\
\hline & FEM & 105.63 & 135.32 & 98.7 & 101.09 & 103.62 \\
\hline & Error $(\%)$ & 0.71 & 0.74 & 0.79 & 0.744 & 0.695 \\
\hline \multirow{4}{*}{$\begin{array}{c}\overline{A_{66}} \\
(\mathrm{MN} / \mathrm{m})\end{array}$} & Theoretical & 25.88 & 20.192 & 27.7 & 27.047 & 26.37 \\
\hline & FEM & 25.893 & 20.195 & 27.7 & 27.063 & 26.412 \\
\hline & Error (\%) & 0.05 & 0.015 & 0 & 0.06 & 0.145 \\
\hline & & Trapezoidal & Rectangular & Toothed & Sinusoidal & Circular \\
\hline \multirow{3}{*}{$\begin{array}{c}\overline{D_{11}} \\
(\mathrm{~N} \cdot \mathrm{m})\end{array}$} & Theoretical & 89.71 & 70 & 96 & 93.763 & 91.43 \\
\hline & FEM & 96.88 & 75.691 & 103 & 101.15 & 98.804 \\
\hline & Error $(\%)$ & 7.4 & 7.52 & 7.08 & 7.3 & 7.46 \\
\hline \multirow{3}{*}{$\begin{array}{c}\overline{D_{12}} \\
(\mathrm{~N} \cdot \mathrm{m})\end{array}$} & Theoretical & 26.91 & 21 & 28.8 & 28.13 & 27.43 \\
\hline & FEM & 29.573 & 23.077 & 31.7 & 30.914 & 30.149 \\
\hline & Error $(\%)$ & 8.99 & 9.00 & 9.00 & 9.00 & 9.00 \\
\hline \multirow{3}{*}{$\begin{array}{c}\overline{D_{22}} \\
(\mathrm{kN} \cdot \mathrm{m})\end{array}$} & Theoretical & 12.95 & 22.792 & 7.35 & 10.548 & 11.82 \\
\hline & FEM & 13.16 & 23.132 & 7.53 & 10.631 & 12.227 \\
\hline & Error $(\%)$ & 1.6 & 1.47 & 2.48 & 0.16 & 3.32 \\
\hline \multirow{3}{*}{$\begin{array}{c}\overline{D_{66}} \\
(\mathrm{~N} \cdot \mathrm{m})\end{array}$} & Theoretical & 53.78 & 68.923 & 50.2 & 51.455 & 52.769 \\
\hline & FEM & 54.51 & 69 & 51.6 & 53.265 & 54.43 \\
\hline & Error $(\%)$ & 1.34 & 0.11 & 2.58 & 3.4 & 3.05 \\
\hline
\end{tabular}

\section{Displacement Analysis}

Displacement of simply supported orthotropic plate under uniformly distributed load in Ref. [14]

$$
w=\frac{16 q}{\pi^{6}} \frac{\sum_{m=1,3,5}^{\infty} \sum_{n=1,3,5}^{\infty} \frac{1}{m n} \sin \frac{m \pi x}{a} \sin \frac{n \pi y}{b}}{D_{11}\left(\frac{m}{a}\right)^{4}+2\left(D_{12}+2 D_{66}\right)\left(\frac{m}{b}\right)^{2}\left(\frac{n}{b}\right)^{2}+D_{22}\left(\frac{n}{b}\right)^{4}}
$$

The parametric study was conducted by ANSYS finite element software. Corrugated structures are modeled with elastic shell element SHELL63. 3-D structural surface effect element SURF154 is used for uniformly distributed load applications in corrugated structure analysis. The length of both sides of the orthotropic plate is $1 \mathrm{~m}$, and the geometry parameter of corrugation is the same as mentioned before; the uniformly distributed load is $69 \mathrm{kN} / \mathrm{m}^{2}$. Displacement of simply supported orthotropic plate under uniformly distributed load can be calculated from Eq.(2), which is compared with the corrugated structure FEA results, as shown in Figs.3-4.

From Figs.3-4, it is obvious that corrugated structures can be replaced by orthotropic plates with equivalent stiffness properties, while analysis of the displacement. 


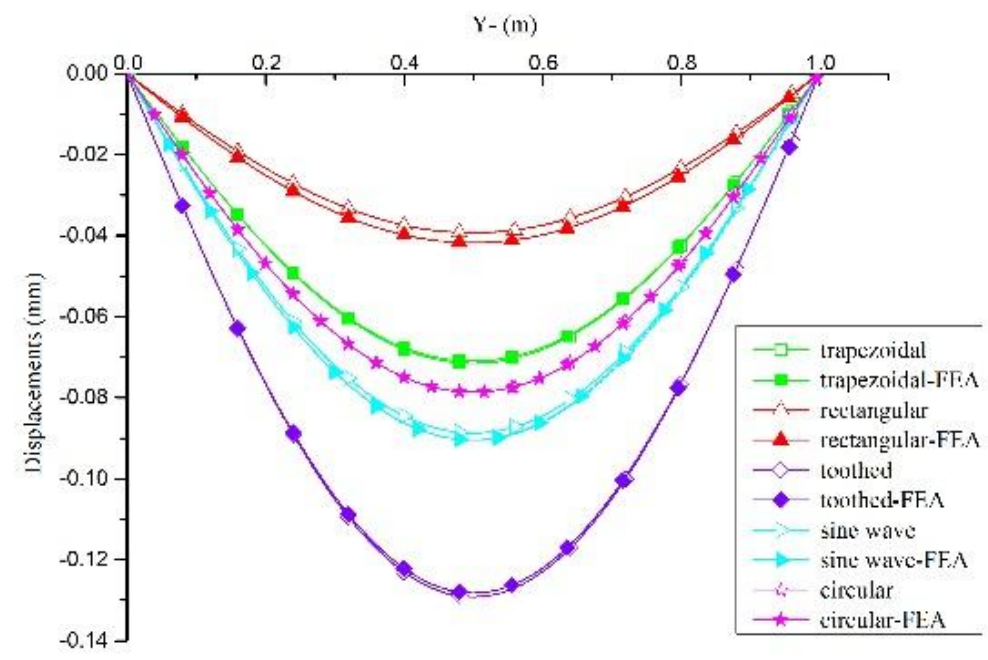

Fig.3 Displacements along Centre Line in X Direction

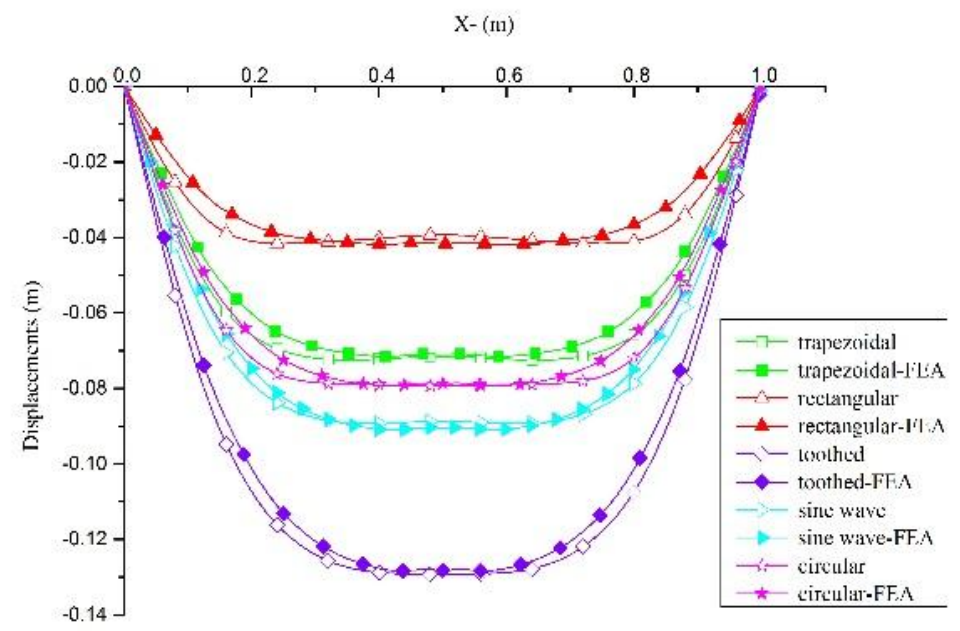

Fig.4 Displacements along Centre Line in Y Direction

\section{Modal Analysis}

Natural frequencies of simply supported orthotropic plate under uniformly distributed load in Ref. $^{[14]}$

$$
\omega^{2}=\frac{\pi^{4}}{\rho}\left[D_{11}\left(\frac{m}{a}\right)^{4}+2\left(D_{12}+2 D_{66}\right)\left(\frac{m}{b}\right)^{2}\left(\frac{n}{b}\right)^{2}+D_{22}\left(\frac{n}{b}\right)^{4}\right]
$$

Natural frequencies of simply supported orthotropic plate under uniformly distributed load can be calculated from Eq. (3), which are compared with the corrugated structure FEA results, as shown in Table 3.

As shown in Table 3, natural frequencies of equivalent orthotropic plates are close to that of corrugated structures; $\mathrm{n}$ maintains 1 in the first five natural frequencies, as bending stiffness $D_{11}$ is much smaller than $D_{22}$. 
Tab.3 Natural Frequencies for Simply Supported Corrugated Structures

\begin{tabular}{|c|c|c|c|c|c|c|}
\hline & $(\mathrm{m}, \mathrm{n})$ & $1(1,1)$ & $2(2,1)$ & $3(3,1)$ & $4(4,1)$ & $5(5,1)$ \\
\hline \multirow{4}{*}{ Trapezoidal } & Theoretical & 28.9419 & 31.1947 & 37.7459 & 50.3124 & 69.0446 \\
\cline { 2 - 7 } & FEM & 29.299 & 32.824 & 41.144 & 55.397 & 75.432 \\
\cline { 2 - 7 } & Error (\%) & $1.2188 \%$ & $4.9637 \%$ & $8.2590 \%$ & $9.1785 \%$ & $8.4678 \%$ \\
\hline \multirow{4}{*}{ Rectangular } & Theoretical & 33.7403 & 35.1679 & 39.2206 & 47.4247 & 60.4822 \\
\cline { 2 - 7 } & FEM & 34.034 & 37.083 & 43.491 & 54.104 & 69.039 \\
\cline { 2 - 7 } & Error (\%) & $0.8630 \%$ & $5.1644 \%$ & $9.8190 \%$ & $12.3453 \%$ & $12.3942 \%$ \\
\hline \multirow{4}{*}{ Sinusoidal } & Theoretical & 22.0167 & 25.8511 & 34.2932 & 49.2996 & 70.5347 \\
\cline { 2 - 7 } & FEM & 23.177 & 27.207 & 36.936 & 53.192 & 75.544 \\
\cline { 2 - 7 } & Error $(\%)$ & $5.0063 \%$ & $4.9836 \%$ & $7.1551 \%$ & $7.3176 \%$ & $6.6310 \%$ \\
\cline { 2 - 7 } & Theoretical & 26.7825 & 29.3522 & 36.7244 & 50.4856 & 70.5692 \\
\cline { 2 - 7 } & FEM & 26.996 & 30.758 & 39.764 & 55.075 & 76.405 \\
\hline \multirow{4}{*}{ Circular } & Theoretical & 27.9488 & 30.3362 & 37.2398 & 50.3247 & 69.6439 \\
\cline { 2 - 7 } & FEM & 28.49 & 32.147 & 40.788 & 55.51 & 76.107 \\
\cline { 2 - 7 } & Error $(\%)$ & $1.8996 \%$ & $5.6329 \%$ & $8.6991 \%$ & $9.3412 \%$ & $8.4921 \%$ \\
\hline
\end{tabular}

\section{Conclusions}

This paper uses equivalent stiffness method to replace corrugated structures by orthotropic plates. After equivalent stiffnesses are obtained, deflections and modals of simply supported orthotropic plates can be analyzed theoretically, which are compared with finite element solutions modeled with corrugated structures. Finally, it is clearly that corrugated structures can be replaced by an orthotropic plate whit equivalent stiffness in deflection and modal analysis.

\section{Acknowledgement}

The authors gratefully acknowledge the financial support provided by the Science and Technology Scheme of Guangzhou City (No. 201510010013), the Science and Technology Scheme of Guangdong Province (No. 2012A030200003) and the National Natural Science Foundation of China (No.11032005).

\section{References}

[1] Briassoulis, D., Equivalent orthotropic properties of corrugated sheets. Computers \& Structures, 1986. 23(2): pp. 129-138.

[2] McFarland, D.E., An investigation of the static stability of corrugated rectangular plates loaded in pure shear, 1967, University of Kansas: Lawrence, KS.

[3] Asokendu Samanta, M.M., Finite element static and dynamic analyses of folded plates. Engineering Structures, 1999. 21: pp. 277-287. 
[4] Tomohiro Yokozeki, S.-i.T., Toshio Ogasawara, Takashi Ishikawa, Mechanical properties of corrugated composites for candidate materials of flexible wing structures. Composites: Part A 2006. 37: pp. 1578-1586.

[5] Peng, L.X., Liew, K.M., Kitipornchai, S., Analysis of stiffened corrugated plates based on the FSDT via the mesh-free method. International Journal of Mechanical Sciences, 2007. 49(3): pp. 364-378.

[6] K.M. Liew, L.X.P., S. Kitipornchai, Vibration analysis of corrugated Reissner-Mindlin plates using a mesh-free Galerkin method. International Journal of Mechanical Sciences, 2009. 51: pp. $642-652$.

[7] Liew, K.M., Peng, L.X., Kitipornchai, S., Nonlinear analysis of corrugated plates using a FSDT and a meshfree method. Computer Methods in Applied Mechanics and Engineering, 2007. 196(21-24): pp. 2358-2376.

[8] Kress, G., Winkler, M., Corrugated laminate homogenization model. Composite Structures, 2010. 92(3): pp. 795-810.

[9] Kress, G., Winkler, M., Corrugated laminate analysis: A generalized planestrain problem. Composite Structures, 2011. 93(5): pp. 1493-1504.

[10] Winkler, M., Kress, G., Deformation limits for corrugated cross-ply laminates. Composite Structures, 2010. 92(6): pp. 1458-1468.

[11] Y. Xia, M.I.F., E.I. Saavedra Flores, Equivalent models of corrugated structures. International Journal of Solids and Structures, 2012. 49: pp. 1453-1462.

[12] Giorgio Bartolozzi, N.B., Marco Pierin, Equivalent properties for corrugated cores of sandwich structures: A general analytical method. Composite Structures, 2014. 108: pp. 736-746.

[13] Zheng Ye, V.L.B., Wenbin Yu, An equivalent classical plate model of corrugated structures. International Journal of Solids and Structures, 2014. 51: pp. 2073-2083.

[14] Shen Guanlin, Hu Gengkai.Mechanics of composite materials[M].Peking: Tsinghua University Press, 2006. (in Chinese) 